\title{
Bilateral Cardiac Sympathetic Denervation on an ECMO patient for Refractory Ventricular arrhythmia: A Case Report.
}

\author{
Akram Farran $^{1}$, Daniel Farinas Lugo ${ }^{2}$, and Joseph Boyer ${ }^{2}$ \\ ${ }^{1}$ Florida State University College of Medicine \\ ${ }^{2}$ AdventHealth Orlando
}

September 25, 2021

\begin{abstract}
Genetic syndromes such as Brugada syndrome can lead to lethal ventricular arrhythmias. Cardiac Sympathetic Denervation has been shown to be effective in ameliorating refractory ventricular arrhythmias. We present a 33-year-old black female with a past medical history of Brugada syndrome with an implantable cardiac defibrillator (ICD), who presented with refractory ventricular tachycardia/atrial fibrillation leading to cardiogenic shock, requiring Extracorporeal membrane oxygenation (ECMO). The patient subsequently underwent bilateral stellate ganglion sympathetic denervation in the setting of refractory ventricular arrhythmias. We present this case report to showcase that thoracoscopic bilateral cardiac sympathetic denervation can be an effective definitive treatment option for ventricular arrhythmias refractory to medical management.
\end{abstract}

\section{Introduction:}

Sudden cardiac death is thought to be caused by ventricular arrhythmias in $75 \%$ to $80 \%$ of cases, resulting in 184,000 to 450,000 deaths in the United States per year [1]. Ventricular arrhythmias are most commonly due to myocardial infarction or cardiomyopathy. Genetic Syndromes such as Brugada Syndrome or catecholaminergic-related polymorphic ventricular tachycardia (CRPVT), are common causes of ventricular arrhythmias but these typically occur in younger patients [2]. An electrical storm (ES) is defined as at least three episodes of ventricular arrhythmias including ventricular tachycardia (VT) or ventricular fibrillation (VF) in 24 hours, significantly increasing risk of heart failure and mortality. An ECMO circuit uses a modified cardiopulmonary machine to support patients and allow time for their cardiac and/or pulmonary function to improve.

Multiple treatments for ES have been proposed, including sympathetic blockade with beta-blockers (BB), anti-arrhythmics, and catheter ablation but due to drug resistance and refractory arrhythmias, other methods have been proposed. Stellate ganglion blockade (SGB), and Cardiac Sympathetic Denervation (CSD) have been attempted in instances of medically refractory ES [4]. We present a patient with Brugada Syndrome and refractory ventricular arrhythmia on ECMO who underwent Video-assisted thoracoscopic surgery (VATS) and Bilateral Cardiac Sympathetic Denervation (BCSD)

\section{Case Presentation:}

Our patient was a 33-year-old black female with a past medical history of Brugada syndrome, she had a St Jude Implantable cardiac defibrillator (ICD) implanted in 2009. Despite her ICD placement, subsequent Radiofrequency ablation (RF) ablation in 2013 and quinidine therapy she remained with persistent atrial fibrillation. She presented to the Emergency Department (ED) with complaints of midsternal non-exertional chest pain and shortness of breath. Initial EKG demonstrated atrial fibrillation with controlled ventricular rate and inferior lateral T-wave inversions. Troponin levels were negative. The patient subsequently went into pulseless VT and despite attempted ablation, she remained in VT. She received multiple rounds of CPR 
with defibrillation until return of spontaneous circulation (ROSC) was achieved. The patient was intubated and placed on ECMO via right femoral access. Cardiothoracic Surgery was consulted to perform a BCSD.

\section{Procedure:}

The patient was brought to the operating room. After insertion of a camera port and 2 additional working ports in the right chest, the right sided sympathetic trunk and stellate ganglia were identified. The sympathetic trunk along with the inferior portion of the stellate ganglia were mobilized at the level of T1-T4 and were then divided using electrocautery. A lateral extension was done to dissect the nerves of Kuntz. The surgical procedure was then repeated on the left side. Bilateral chest tubes were placed. The patient tolerated the procedure well.

Following the procedure, the patient did not experience any episodes of VT and she subsequently remained in sinus rhythm. A postoperative echo demonstrated improved ejection fraction of $40 \%$ and the patient was decannulated from ECMO 3 days after the operation. The patient continued to improve and was weaning off her blood pressure support. She remained in sinus rhythm and on anticoagulation due to right lower extremity DVT. Unfortunately, the patient experienced sudden onset hemorrhagic shock from a spontaneous liver laceration several weeks after the operation and expired.

\section{Discussion:}

Brugada Syndrome is an inherited cardiac electrical disorder occurring in the absence of obvious structural heart disease, leading to sudden cardiac death due to polymorphic ventricular tachycardia. Patients with Brugada Syndrome can suffer from electrical storms, which causes excess catecholamine release from sympathetic fibers leading to sympathetic hyperactivity outflow to the heart resulting in potentially fatal arrhythmias [3] [4] [5].

Studies have shown benefit in restoring systemic circulation using ECMO in patients with cardiogenic shock related to ES and hemodynamic instability in ventricular arrhythmias [3]. ECMO support can stabilize the patient in order to facilitate future surgical interventions in more favorable hemodynamic conditions. Despite documented benefits, there appears to be an increase in mortality that is directly proportional to length of time on ECMO [6]. This supports the use of ECMO as a bridge to intervention rather than for prolonged survival in patients with cardiogenic shock.

Stellate Ganglion Block is traditionally performed by injecting local anesthetics percutaneously to the stellate ganglion. Though SGB has been shown to be effective, its therapeutic effectiveness and duration is variable depending on the type of anesthesia and method of administration [7].

CSD describes the surgical resection of the majority or the lower half of the stellate ganglion as well as the sympathetic chains from T1-T4. CSD interrupts the major source of norepinephrine

released to the heart and has multiple antiarrhythmic effects including increasing the threshold for ventricular fibrillation and increasing ventricular refractory period [8]. When standard medical treatments fail, Wilde et al. demonstrated the effectiveness of left sided CSD in patients who suffered from catecholaminergic polymorphic VT [8]. Symptoms were controlled for up to 20 years postoperatively in some patients, although ventricular arrhythmias did occur at high workloads. Left Cardiac Sympathetic Denervation (LCSD) is more commonly performed, but Bilateral Cardiac Sympathetic Denervation (BCSD) or right sided CSD (RCSD) performed as an adjunct to a previously failed LCSD has also been done with promising results [9] [10]. In canine studies comparing left, right, or bilateral sympathectomy, the most profound anti-arrhythmic effects were seen with bilateral sympathectomy [11]. Despite being more invasive, bilateral CSD appears to provide a more effective therapeutic intervention. It can prevent catecholamine surges and decrease the likelihood of life-threatening arrhythmias that are refractory to defibrillation.

\section{Conclusion :}

We present this case in order to review a rare condition and suggest that in cases when routine management of ES, such as defibrillation, anti-arrhythmic therapies, and ablation, fail to control life-threatening ventricular 
arrhythmias, ECMO support followed by CSD, performed by an experienced cardiothoracic surgeon, can be an effective and definitive treatment option.

\section{Sources:}

1. Chugh SS, Reinier K, Teodorescu C, et al. Epidemiology of sudden cardiac death: clinical and research implications. Prog Cardiovasc Dis . 2008;51(3):213-228. doi:10.1016/j.pcad.2008.06.003

2. Prystowsky EN, Padanilam BJ, Joshi S, Fogel RI. Ventricular arrhythmias in the absence of structural heart disease. J Am Coll Cardiol. 2012 May 15;59(20):1733-44. doi: 10.1016/j.jacc.2012.01.036. PMID: 22575310.

3. Aitor Uribarri, Loreto Bravo, Javier Jimenez-Candil, Javier Martin-Moreiras, Eduardo Villacorta, Pedro L Sanchez, Percutaneous extracorporeal membrane oxygenation in electrical storm: five case reports addressing efficacy, transferring allowance or radiofrequency ablation support, European Heart Journal. Acute Cardiovascular Care, Volume 7, Issue 5, 1 August 2018, Pages 484-489.

4. Asangmbeng NV, Okoduwa D, James EA. Stellate Ganglion Blockade and Left Cardiac Sympathetic Denervation With Left Stellate Ganglionectomy in a Patient With Refractory Electrical Storm: A Case Report. Cureus . 2020;12(7):e9098. Published 2020 Jul 9. doi:10.7759/cureus.9098

5. Maury P, Hocini M, Haïssaguerre M. Electrical storms in Brugada syndrome: review of pharmacologic and ablative therapeutic options. Indian Pacing Electrophysiol J . 2005;5(1):25-34. Published 2005 Jan 1.

6. Chen CY, Tsai J, Hsu TY, et al. ECMO Used in a Refractory Ventricular Tachycardia and Ventricular Fibrillation Patient: A National Case-Control Study. Medicine (Baltimore) . 2016;95(13):e3204. doi:10.1097/MD.0000000000003204

7. Meng L, Tseng CH, Shivkumar K, Ajijola O. Efficacy of Stellate Ganglion Blockade in Managing Electrical Storm: A Systematic Review. JACC Clin Electrophysiol . 2017;3(9):942-949. doi:10.1016/j.jacep.2017.06.006

8. Wilde AA, Bhuiyan ZA, Crotti L, Facchini M, De Ferrari GM, Paul T, Ferrandi C, Koolbergen DR, Odero A, Schwartz PJ. Left cardiac sympathetic denervation for catecholaminergic polymorphic ventricular tachycardia. N Engl J Med. 2008 May 8;358(19):2024-9. doi: 10.1056/NEJMoa0708006. PMID: 18463378.

9. Ajijola OA, Lellouche N, Bourke T, et al. Bilateral cardiac sympathetic denervation for the management of electrical storm. J Am Coll Cardiol . 2012;59(1):91-92. doi:10.1016/j.jacc.2011.09.043

10. Assis FR, Sharma A, Shah R, et al.Long-Term Outcomes of Bilateral Cardiac Sympathetic Denervation for Refractory Ventricular Tachycardia. JACC Clin Electrophysiol 2021;7:463-470.

11. Brooks WW, Verrier RL, Lown B. Influence of vagal tone on sympathectomy-induced changes in ventricular electrical stability. Am J Physiol. 1978;234:H503-H507. 